\title{
COMMENTAIRES A PROPOS DU SEMINAIRE SUR LES EAUX CONTINENTALES 18-19 Novembre 1975 - AMIENS
}

\author{
Jacques ARRIGNON \\ Ingénieur I.A.N. \\ Vice-Président de I'Institut d'Ecologie \\ du Bassin de la SOMME
}

\section{INTRODUCTION}

L'Institut d'Ecologie du Bassin de la Somme a été fondé en 1974 dans le but de contribuer à l'étude de l'influence des activités humaines sur les milieux naturels du Bassin de la Somme et, ce faisant, d'éclairer les autorités responsables dans leur choix d'aménagements en vue de la sauvegarde de ces milieux en tant que facteurs d'équilibre.

Antérieurement à cette fondation, un Groupe de travail avait, en 1973 et en 1974, dressé le bilan des actions de recherches, d'ètudes et d'expérimentations en cours et projetées dans le Bassin de la Somme, à l'effet de les coordonnet et de mettre en commun certaines connaissances et certains moyens.

La confrontation des hommes, des idées et des travaux, fructueuse au niveau régional, a incité l'I.E.B.S. à organiser une réunion de chercheurs, techniciens et aménageurs portant leurs efforts sur des problèmes analogues dans des bassins fluviaux de mème type, se répartissant ainsi dans la zone sous influence maritime du Nord-Ouest Européen.

Cette réunion a été conçue sous la forme d'un Séminaire, plutôt que sous celles d'un Symposium ou d'un Colloque, pour les raisons parfaitement détail- 
lées, dans son allocution introductive, par le Professeur Henri HOESTLANDT, Président de I'I.E.B.S.

Un Séminaire constitue en quelque sorte pour les jeunes chercheurs et les jeunes ingénieurs, non seulement l'occasion de s'exprimer et de prendre les contacts nécessaires à la progression de leurs connaissances et de leurs travaux, mais un véritable banc d'essais avant d'affronter des assises plus rigoureuses au hasard de leur carrière professionnelle.

II convient donc de les conforter dans leur désir de présenter des communications courtes, sur un sujet précis ou sur une synthèse bien comprise et bien bâtie, exprimée en langage clair et concis, d'une voie distincte en ayant le souci d'éclairer et d'agrémenter le texte par des illustrations bien choisies et convenablement réalisées, ou bien de commenter sobrement mais suffisamment une architecture d'illustrations, conçue en fonction du sujet à traiter.

En dehors de ces contingences le but d'un Séminaire est de faire le point sur le thème choisi, au travers des travaux des diverses disciplines livrés à la connaissance et à la discussion de chacun.

Ces travaux ont été classés et présentés en tenant compte d'une certaine progression allant de thèmes relatifs à l'étude du milieu aquatique et des indicateurs biologiques, exposés au cours de la première journée, à celle des bassins versants et de leur aménagement par l'Homme, au cours de la seconde journée. II n'est pas douteux que l'analyse des seuls titres a conduit à la conception d'un pré-programme imparfait, imparfaitement corrigé par l'analyse suivante portant sur les résumés: quelques transferts de communications auraient pu être opérés d'une journée à l'autre mais on peut se demander s'il n'y aurait pas eu un déséquilibre fâcheux entre le volume des travaux de la première journée et celui des travaux de la seconde. Par sa nature mème, l'Ecologie n'est pas simple, ni dans son approche, ni dans l'expression de cette approche, ni dans l'homogénéité de cette expression.

L'audition attentive de l'ensemble des communications et des discussions qui ont suivi, les échanges de conversation à cet égard entre bon nombre des participants, portent à penser toutefois que les centres d'intérêts manifestés ont été cohérents et se sont bien intégrés dans l'esprit général du Séminaire et dans les orientations données a a priori " aux deux journées.

\section{CONTENU ET DISCUSSION DES tRAVAUX}

* La dynamique des matières en suspension dans un bief du Lot * a donné l'occasion à $H$. DECAMFS de souligner l'importance de la nature de l'impluvium dans le taux d'entrainement des sédiments, à débit comparable des tributaires, de même que celle des débits limites entrainant dans le cours principal l'arrachage des sédiments et leur décantation.

La discussion a fait apparaitre certaines incertitudes liées à la difficulté d'apprécier certains phénomènes hydrauliques, notamment dans la zone lentique des bordures et des mouilles, phènomènes que l'on pressent dans les divagations de la Somme Supérieure ainsi que dans d'autres cours d'eau de même régime et de même allure. Ces inquiétudes doivent motiver des recherches d'hydraulique et de sèdimentologie fines, liées à l'hydrobiologie et impliquer ainsi d'autres collaborations dans les équipes d'hydrobiologistes. 
Le rôle des sédiments comme pièges à phosphore, évoqué par le $\mathrm{Dr}$ GOLTERMAN dans les nouveaux lacs néerlandais n'est pas sans rapport avec certaines anomalies dans le développement algal et dans la pauvreté de certains milieux lentiques du Nord de la France dont le pouvoir auto-épurateur est freiné ainsi qu'a pu le constater J. LEGAGNEUR en analysant l'évolution hydrobiologique de la Haute Somme et en s'inquiétant d'une disparition inexplicable de certains sels tels que les nitrates.

Les effets de lagunage artificiel étudiés par M.C. DELANNOY-HOEDTS sur le plan algal auraient mérité un complément d'information en ce qui concerne l'intervention concomitante du zooplancton et on a pu regretter à cet égard l'absence de N. ANGELI qui avait travaillé sur des sites identiques, sinon sur le même site. La durée du transit de l'eau dans les bassins ou étangs de lagunage doit certainement être liée à la durée du temps de vie des organismes consommateurs et, comme l'ont fait remarquer B. DUSSART, R. POURRIOT et J.-P. DESCY, l'étude de la dynamique d'une espèce, dans un milieu donné, est probablement plus révélatrice que la seule notion d'absence ou de présence.

La notion spatiale de support, de substrat, doit elle-même être précisée et nous avons noté, à cet égard, l'intéressante remarque faite par $M$. LOQUET et par J.-P. DUPONT à propos des terriers de Nereis qui, pour une projection de $1 \mathrm{~m}^{2}$ au sol, occupent une surface développée de $6 \mathrm{~m}^{2}$, de même que celle relative à l'importante surface périphytique des briophytes pour la même surface projetée, de $1 \mathrm{~m}^{2}$ (A. EMPAIN) : on est plutôt amené à parler de biovolume au mètre carré et c'est ce que font depuis déjà de nombreuses années les hydrobiologistes d'application, entreprenant des études piscicoles de rivières, en appréciant les caractéristiques morphodynamiques, la nature et la répartition des couvertures végétales dans les cours d'eau: l'approche quantitative ne peut avoir de sens s'il en est différemment.

Le type d'études concernant un grand cours d'eau navigué, la Sambre, a conduit ses auteurs, et notamment R. KAISER et J.-C. MICHA, à rechercher des méthodes d'échantillonnage permettant de mieux connaitre la dynamique de la vie sur et dans les substrats des fonds ainsi que dans l'échappement spatial du cours d'eau. Nous avons noté l'identité des vues au sujet de l'influence de la turbulence due à la navigation sur l'instabilité, et même sur la disparition de certaines espèces benthiques, constatées également dans l'Aisne naviguée et dans les canaux de Saint-Omer. Des constatations analogues dans la Tamise ont été relatées par A.-D. BERRIE.

De nombreux chercheurs se sont penchès sur le rôle de tel ou tel organisme comme indicateur de la qualité des eaux et ont essayé de vérifier, voire de compléter, tel G. VANHOOREN, les différentes échelles de saprobité. On se rend compte que cette approche est toujours aussi délicate en raison notamment de la diversité des milieux auxquels on veut les appliquer et combien doivent être prudentes les extrapolations : les échelles de saprobité, l'établissement des indices biotiques de TUFFERY et VERNEAUX, le calcul des indices de diversité, même confrontés, ne livrent pas toujours l'identité d'une situation et encore moins son évolution, notamment dans les milieux lentiques complexes. A cet égard, l'étude d'une chaine alimentaire complète est peut-être plus révélatrice, les études d'écologie piscicole remontant jusqu'au terme final qu'est la dynamique des populations de poissons le sont sûrement davantage mais leur mise en œuvre est freinée par leur lourdeur logistique et par leur coût élevé : leur emploi est donc subordonné à un état d'urgence concernant l'avenir du tronçon ou de l'ensemble des cours d'eau à étudier. 


\section{C'est précisément vers le Bassin que l'on a plutôt tendance à porter désor- mais l'ensemble des efforts d'investigation.}

Les communications qui s'y sont appliquées ont été pour l'essentiel des communications de groupes de travail, car il est difficile d'imaginer autrement une approche aux implications aussi nombreuses. Elles ont montré l'incidence des activités humaines dans les bassins versants, qu'elles soient anciennes et traditionnelles en Haute Somme, dans le Sud de l'Angleterre, dans la vallée de la Bresle, dans l'Entre Deux Mers et dans les Marais d'Arleux, ou récentes comme dans le Bassin de la Tamise, de la Sambre, de la Somme en aval d'Amiens, évidentes ou subtiles, par suite de la modification des couvertures végétales, des pratiques culturales dans les bassins versants des petits cours d'eau côtiers, entrainant la disparition d'un impluvium d'absorption et l'apparition d'un impluvium de ruissellement. Les communications de A.-D. BERRIE, F. GROSS, M. LADLE, T.-T. MACAN et Ph. MEROT ont été éloquentes et intéressantes à ce point de vue.

Un changement du régime fluvial et un basculement des dominances ont été bien mis en évidence dans la région des hortillonnages par P.-J. LE MORVAN en ce qui concerne l'avifaune et, dans le bassin du Scorff, en Bretagne, grâce aux travaux de M. THIBAULT et de son équipe, qui, passant de l'investigation à l'action rénovatrice, ont montré la voie des réaménagements souhaitables.

Ces entreprises sont malheureusement parfois sujettes à des impondérables très pondéreux, telle l'apparition du syndrome mycosique qui a décimé le cheptel salmonicole de la Bresle, comme l'a montré J.-J. DEMARS, et de nombreux autres cours d'eau français, ou encore, à un autre titre, la manifestation de certaines toxicités insolites en pisciculture, dues à la dégradation, par exemple, d'une couche très superficielle des parois des bassins d'élevage en résine synthétique, selon les travaux de J. DEQUIDT, F. ERB, J.-C. CAZIN et J. POMMERY. Les chercheurs des Facultés de Médecine et de Pharmacie viennent ainsi s'intégrer aux équipes d'écologistes pour aborder l'étude d'orientations très spéciales et souvent fondamentales (J. GORIN et P. HARICHAUX), à l'opposé mais en complémentarité avec les enquêtes effectuées par les géographes, économistes et sociologues.

L'action des usagers de l'eau gagne en effet à être mieux connue dans les bassins versants dont certains sont très sollicitès pour l'alimentation humaine, tels ceux de la Tamise (A.-D. BERRIE) et de Saint-Omer. Elle est même à contrôler, voire à réguler, du moins en ce qui concerne les activités de détente et de loisirs qu'il s'agisse de la chasse, de la pêche ou de la promenade dont l'incidence potentielle a été étudiée par E. VIGNEUX sur les étangs d'Arleux dans le Nord.

L'effort de pêche doit correspondre à la productivité des collections d'eau ou, à la limite, correspondre quantitativement et qualitativement à des apports extérieurs réguliers, quand l'eau n'est plus qu'un simple support, ce qui malheureusement est encore très fréquent dans les bassins fluviaux du Nord-Ouest de l'Europe. L'intensité, la fréquence, l'amplitude et la périodicité de cet effort de pêche doivent être connus au travers d'enquêtes socio-économiques préalables aux propositions de protection et de réaménagement de ces bassins comme l'ont montré Y. ARRIGNON pour la cuvette de Saint-Omer dans le Pas-de-Calais, M. THIBAULT et $P$. RAINELLI pour le bassin du Scorff. Le zonage des milieux fragiles et menacés par les activités humaines, proposé par F. VIGNON et I.-R. WATTEZ, suppose la livraison, au pouvoir de décision, d'un outil évolutif et perfectible mais fait craindre à de nombreux intervenants que ce caractère même ne soit cause de report et d'ajournement dans les décisions à prendre. 


\section{CONCLUSION}

La présentation des travaux et les confrontations d'idées de ce Séminaire imposent au moins trois remarques:

1) La nécessité de poursuivre les études entreprises dans les différents secteurs en s'entourant de toutes les garanties possibles et en faisant appel aux collaborations les plus souhaitables. L'ouverture doit être la règle, le travail a en solo * doit faire place au travail en équipe dans la plupart des cas, ceux des spécialistes très spécialisés exceptés. L'artisanat en hydrobiologie et en écologie peut conduire en effet à des conclusions aberrantes.

2) La nécessité de rapprocher les spécialistes des différentes disciplines touchant de près ou de loin l'étude des activités humaines dans un bassin versant, le bassin versant devant être considéré comme une unité écologique.

3) La nécessité de rapprocher les chercheurs, les concepteurs et les décideurs, de fournir à ces derniers les synthèses les plus objectives qui soient avant que des initiatives très agressives soient prises à l'égard de tel ou tel bassin. Ce rapprochement doit conduire à une meilleure information et de part et d'autre, à l'abandon de prises de position hâtives et gratuites.

La présence active lors de ce Séminaire, d'Ingénieurs, d'Aménageurs, de responsables des ressources aquatiques des Services Régionaux d'Aménagement des Eaux de Haute-Normandie et de Picardie, de l'Agence Financière du Bassin Artois-Picardie, du Secrétariat Général et de toutes les Régions Piscicoles du Conseil Supérieur de la Pêche est un indice certain de l'évolution des rapports et d'une prise de conscience à l'échelon national.

La participation de nos collègues étrangers, belges, britanniques, néerlandais, montre que les relations internationales, recommandées par IUNESCO dans son programme 5 * Incidence des activités humaines sur les milieux aquatıques et palustres", sont réelles et bien établies.

Tels étaient les objectifs du Séminaire sur les Eaux Continentales du NordOuest Européen, organisé par l'Institut d'Ecologie du Bassin de la Somme. 